\title{
miR-21 regulates vascular smooth muscle cell function in arteriosclerosis obliterans of lower extremities through AKT and ERK1/2 pathways
}

\author{
Shuichuan Huang ${ }^{1}$, Tuo Xü ${ }^{2}$ Xianying Huang ${ }^{1}$, Siyi Li ${ }^{2}$, Wenyi Qin², Weijie Chen², Zhi Zhang ${ }^{2}$
}

${ }^{1}$ Department of Vascular Surgery, Nanfang Hospital, Southern Medical University, Guangzhou, China

${ }^{2}$ Department of Vascular Surgery, Affiliated Hospital of Guangdong Medical

University, Zhanjiang, China

Submitted: 30 May 2018

Accepted: 2 September 2018

Arch Med Sci 2019; 15 (6): 1490-1497

DOI: https://doi.org/10.5114/aoms.2018.78885

Copyright @ 2018 Termedia \& Banach

\section{Abstract}

Introduction: Arteriosclerosis obliterans (ASO) is a disease that affects the lower extremities. The mechanism of ASO is associated with the proliferation and migration of vascular smooth muscle cells (VSMCs). miR-21 plays a key role in various biological processes of the cardiovascular system, associated with the proliferation, migration and apoptosis of VSMCs. It is unclear, however, if miR-21 is involved in the regulation of ASO.

Material and methods: Human aortic smooth muscle cells (HASMCs) were transfected with miR-21 mimics and co-treated with protein kinase B (AKT) or a mitogen-activated protein kinase (ERK) inhibitor. Expression levels of p-AKT or p-ERK were measured by western blot. Cell apoptosis was assessed using the terminal deoxynucleotidyl transferase dUTP nick end labeling (TUNEL) assay and visualized under a fluorescence microscope. Cell proliferation was monitored by bromodeoxyuridine (BrdU) labeling; cell migration and invasion were determined by the Transwell assay.

Results: miR-21 was upregulated in arteries of ASO, the pathogenesis of which involved the activation of $p$-AKT and $p$-ERK1/2. Inhibition of the AKT or ERK activity was consistent with the attenuation of the miR-21-induced HASMC migration and proliferation. HASMCs co-treated with miR-21 mimics and AKT or ERK inhibitor showed attenuation of the miR-21-induced high elongation ratio.

Conclusions: We demonstrated that the expression of miR-21 in HASMCs could find potential application in cardiac therapy. Inhibition of the activity of AKT or ERK could attenuate miR-21-induced cell proliferation and migration as well as altering morphology of HASMCs. The present study aimed to indicate the potential roles of miR-21 in ASO processes, and the results provided a novel therapeutic approach for treating ASO and new targets for preventing ASO in earlier stages.

Key words: arteriosclerosis obliterans, miR-21, HASMCs, AKT, ERK.

\section{Introduction}

Atherosclerosis, a chronic progressive disease relating the immune or inflammatory response to vascular tissue damage, can affect coronary, cerebral, and lower extremities arteries [1-3]. Arteriosclerosis obliterans (ASO) of the lower extremities represents a spectrum of disorders

\author{
Corresponding author: \\ Zhi Zhang \\ Department \\ of Vascular Surgery \\ Affiliated Hospital \\ of Guangdong \\ Medical University \\ 57 South of Renmindadao \\ 524001 Zhanjiang \\ Guangdong Province, China \\ E-mail: zhangzhifdsa@163.com
}


causing normal limb dysfunction and may be associated with an increased risk for claudication, resting pain and gangrene $[4,5]$. Open surgery, endovascular therapy, and physical therapy (e.g. exercise) are the major methods to cure ASO [6]. Nevertheless, the risk of patients developing post-angioplasty restenosis within 1 year after the these treatments, such as balloon angioplasty procedures and stent implantations, is high [7]. The damage caused by ASO includes a series of highly specific cellular and molecular responses; changes in the phenotype of vascular cells, such as platelets or inflammatory cells, that release growth factors and cytokines to induce vascular smooth muscle cells (VSMCs) are observed after the initial arterial injury [8].

The mechanism of ASO is associated with VSMCs' proliferation and migration, which are stimulated by the inflammatory response $[9,10]$. Significant differences are found in the expression levels of some microRNAs, such as miR-21, miR22, miR-24 and miR-1298, between ASO and normal arteries [11]. Among these microRNAs, miR-21 plays a critical role in various biological processes in the cardiovascular system, regulating heart and vascular function; it is also associated with proliferation, migration and apoptosis of VSMCS $[12,13]$. miR-21 is one of the well-characterized miRNAs: previous research has shown that the expression of miR-21 was remarkably elevated in cardiovascular cells and such overexpression has also been found in other cardiovascular diseases, such as obliterative vascular disease, ventricular hypertrophy and ischemic heart disease [13, 14].

Mitogen-activated protein kinases (MAPKs) were found to be potential mediators in the inflammatory response and atherosclerosis $[15,16]$. Pathways of two important members of the MAPK family, ERK $1 / 2$ and AKT, were proved to regulate multiple biological processes including cell survival, proliferation and glycogen metabolism in VSMCs [17, 18].

Recently studies reported that inhibition of the PI3K/AKT signaling pathway also inhibited atherosclerosis progression $[19,20]$ but enhanced the stability of atherosclerotic plaques [21]. Compared with normal vessel tissues, the tissues from atherosclerotic lesions showed a significant increase in the expression of ERK $1 / 2$ protein $[22,23]$. It is still unclear, however, if miR-21 and ERK and AKT pathways are involved in the regulation of ASO. In this study, we developed an ASO injury rat model to determine whether the expression of miR-21 was stimulated under the ASO condition. A cell model was used to investigate whether AKT and ERK $1 / 2$ pathways were involved in the miR-21-induced proliferation and migration of human aortic smooth muscle cells (HASMCs). We also attempt- ed to determine whether expression of AKT and ERK $1 / 2$ could modulate the miR-21-induced alteration of cell shape in HASMCs.

\section{Material and methods}

\section{Cell culture and cell transfection}

HASMCs were maintained in DMEM medium (Sigma) supplemented with $10 \%$ fetal bovine serum (FBS) (Hyclone), penicillin (100 U/ml) (Sigma) and streptomycin $(100 \mathrm{mg} / \mathrm{ml})$ (Sigma). Cells were incubated at $37^{\circ} \mathrm{C}$ in a humidified atmosphere of $5 \%$ $\mathrm{CO}_{2}$ and $95 \%$ air, and only cells within the subculture passage 4 to 10 were used in subsequent experiments. Cells were grown to $80-90 \%$ confluence. Serum-free medium was replaced and the cells were incubated for $24 \mathrm{~h}$ to reach $90 \%$ confluence to induce quiescence. A miR-21 mimic was synthesized and was transfected into HASMCs using Lipofectamine 2000 (Invitrogen) transfection reagent. Cells were incubated for $6 \mathrm{~h}$ in serum-free medium, which was then replaced with complete growth medium. Transfection was initiated when the cells reached $70-90 \%$ confluence. Cells were incubated at $37^{\circ} \mathrm{C}$ for $2-4$ days and using a microscope to confirm the efficiency of transfection. RNA and protein were extracted and collected after transfection and analyzed by western blot and real-time PCR.

\section{Western blot analysis}

HASMCs or the vessel tissue were lysed and extracted. The protein concentrations were determined by the bicinchoninic acid (BCA) assay (Thermo Fisher Scientific). Protein extract was separated by $10 \%$ or $6 \%$ SDS-PAGE and transferred to a nitrocellulose or polyvinylidene difluoride (PVDF) membrane. After $1 \mathrm{~h}$ incubation in blocking solution, membranes were immersed using primary antibodies (anti-AKT (Abcam), anti-pAKT (Abcam), anti-ERK1/2 (Abcam), anti-pERK1/2 (Abcam)) at $4^{\circ} \mathrm{C}$ overnight. Subsequent to washing with TBST, the membranes were incubated with $1: 5000$ dilution secondary antibodies for $1 \mathrm{~h}$. The membranes were detected with the enhanced chemiluminescence $(E C L)$ system. Density of each band was analyzed using Image J.

\section{Cell apoptosis assays}

Apoptotic cells were detected with a terminal deoxyribonucleotidyl transferase-mediated dUTP-digoxigenin nick-end labeling (TUNEL) assay kit (Abcam). Nuclear morphology was stained by 4,6-diamidino-2-phenylindole (DAPI) staining (Sigma). HASMCs were washed with PBS and fixed with $4 \%$ paraformaldehyde at $4^{\circ} \mathrm{C}$, followed by cell membrane permeabilization with Triton X-100. The apoptosis rate was quantified by counting 
cells with apoptotic nuclei by using a fluorescence microscope.

\section{Quantitative real-time PCR}

HASMCs or the vessel tissues were harvested, and quantitative stem-loop RT-PCR for detection of miR-21 level was determined with SYBR Green Master Mix kits (Thermo Fisher Scientific). The expression of miR-21 was normalized to rRNA U6. Single-strand cDNA was synthesized from total RNAs using a Reverse Transcription System Kit (Promega, Madison, WI).

\section{Immunohistochemistry staining}

The rat normal and ASO arterial tissues were fixed with paraformaldehyde (PFA) in PBS for $30 \mathrm{~min}$. Transverse sections $(10 \mu \mathrm{m})$ of frozen vessels were prepared using coated slides, which were fixed with PFA (4\%). Tissue sections were permeabilized with Triton-X-100 (0.2\%) in PBS for $10 \mathrm{~min}$. Primary and secondary antibodies were applied to the tissue at room temperature for $1 \mathrm{~h}$. The primary antibodies used were as follows: miR-21 (Abcam), AKT (Abcam), ERK1/2 (Abcam) and actin (Abcam). The slides were washed with PBS after co-incubation with each antibody and were then analyzed under a fluorescence microscope. The animal experiments were approved by the Affiliated Hospital of Guangdong Medical University.

\section{Proliferation, invasion and migration assay BrdU incorporation}

HASMCs were transfected with miR-21 mimics or miR-21 inhibitor. Cells were incubated either in the presence or absence of AKT and ERK inhibitors at $37^{\circ} \mathrm{C}$. Cell proliferation was measured at $24 \mathrm{~h}$, $48 \mathrm{~h}$ and $72 \mathrm{~h}$ after transfection with a BrdU assay kit, respectively. The culture medium was removed and replaced with BrdU labeling solution, after which the HASMCs were incubated in the BrdU labeling solution at $37^{\circ} \mathrm{C}$. The cells were fixed with paraformaldehyde and stained with DAPI after washing with PBS, and then measured by immunocytochemistry.

\section{Transwell assay and wound healing assay}

HASMCs were plated with a density of $2 \times 10^{4}$ cells/well in the top compartments inserted into the wells and complete medium $(600 \mu l)$ was added to the lower reservoir chamber. Subsequent to incubation at $37^{\circ} \mathrm{C}$ for $6-24 \mathrm{~h}$, the cells without migration were scraped off whereas the cells that migrated to the bottom side were stained with $10 \%$ Giemsa. The migration ability was expressed by counting the stained cells on the bottom surface.
The wound closure assay was performed to evaluate the ability of the cells to migrate. The HASMCs were plated with a density of $1 \times 10^{6}$ cells/well in 12 -well plates and were then scratched. The average distance that cells moved from the edge of the scratch towards the center was measured after $24 \mathrm{~h}$.

\section{Statistical analysis}

Statistical analysis was performed using GraphPad Prism. Data were reported as means \pm (SEM) from three independent experiments. Statistical analysis was performed using the $t$-test to compare differences between two groups. Multiple group comparisons were analyzed using ANOVA with a post hoc test. $P<0.05$ was considered to indicate a statistically significant result.

\section{Results}

\section{miR-21 expression levels were increased in ASO arteries}

To determine whether miR-21 expressed in the ASO arteries, quantitative RT-PCR was used to measure the expression level of miR-21 in ASO and normal arterial walls. As shown in Figure $1 \mathrm{~A}$, compared with the normal arteries, the miR-21 expression level was significantly increased in the ASO arteries. Subsequently, the expression pattern of miR-21 in different layers of ASO artery was investigated. As shown in Figure $1 \mathrm{~B}$, the expression level of miR-21 in the media of ASO artery was significantly higher than that in the layer of adventitia and intima. Immunofluorescence results confirmed that the expression of miR-21 was situated in the media of the ASO artery (Figure $1 \mathrm{C}$ ). The results indicated that miR-21 was upregulated in the ASO artery.

\section{The expression of AKT and ERK1/2 were aberrant in the ASO arteries}

In order to determine whether expression of AKT and ERK $1 / 2$ was involved in the pathogenesis of ASO, expression levels of p-AKT and p-ERK $1 / 2$ were compared between normal and ASO arteries using western blot. As shown in Figure $1 \mathrm{D}$, the expression levels of $p$-AKT and p-ERK $1 / 2$ were significantly increased in the ASO artery. The results demonstrated that expression of p-AKT and p-ERK1/2 was involved in the pathogenesis of ASO.

\section{Down-regulation of AKT and ERK $1 / 2$ expression inhibited the miR-21-induced proliferation and migration of HASMCs}

To determine whether miR-21 was involved in the regulation of HASMC motility, HASMC was transfected with miR-21 mimics. Quantitative real-time RT-PCR was used to measure the expres- 
A

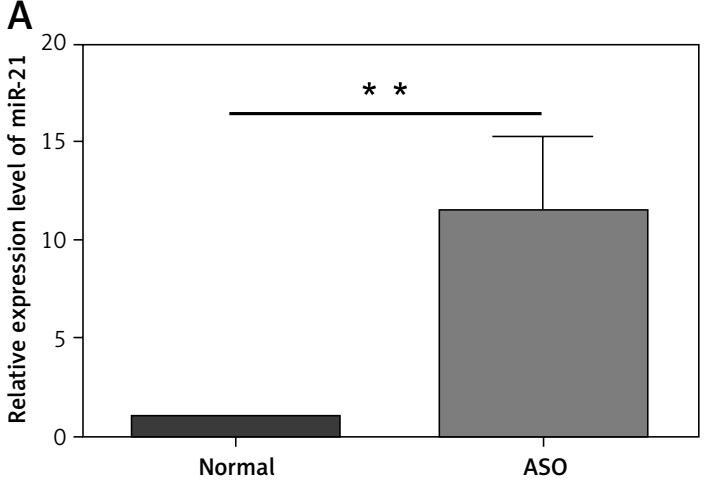

C
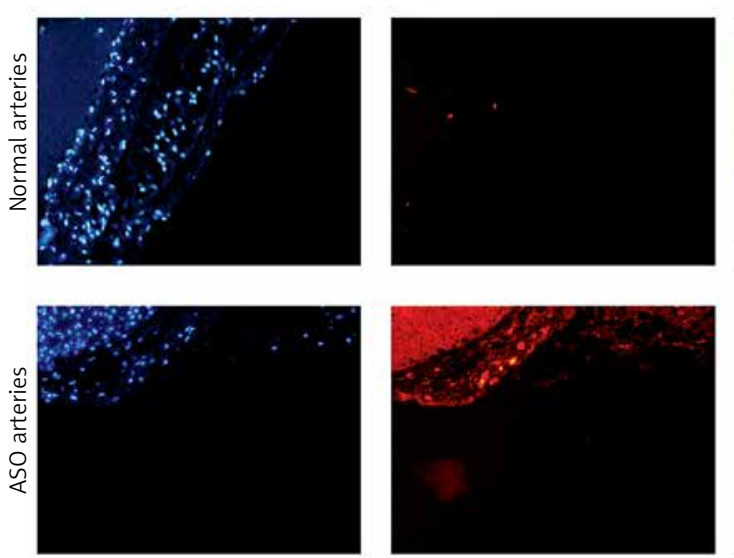

D
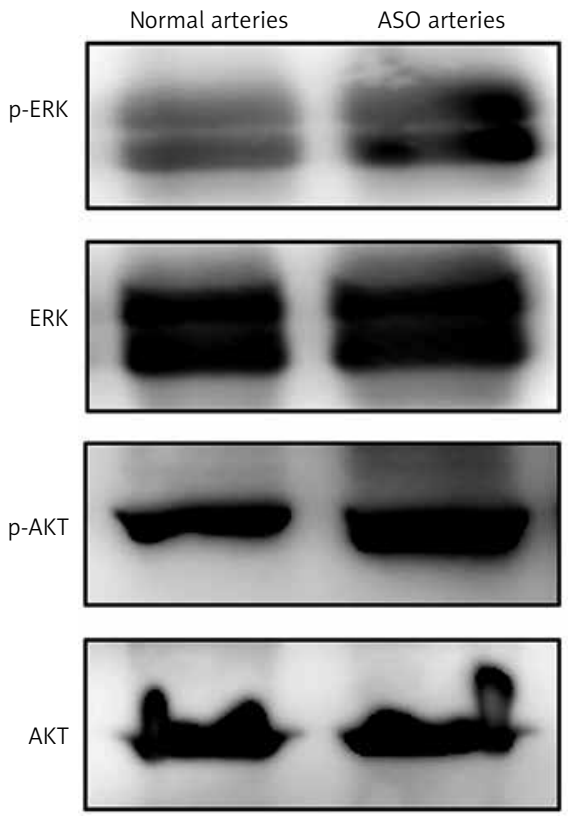

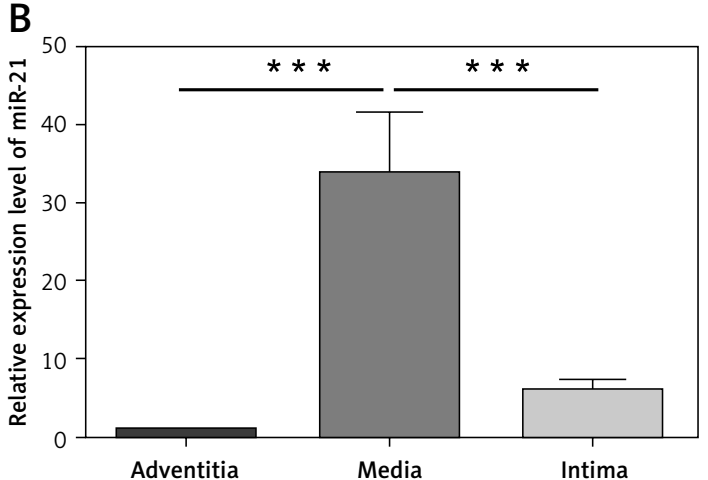

Actin
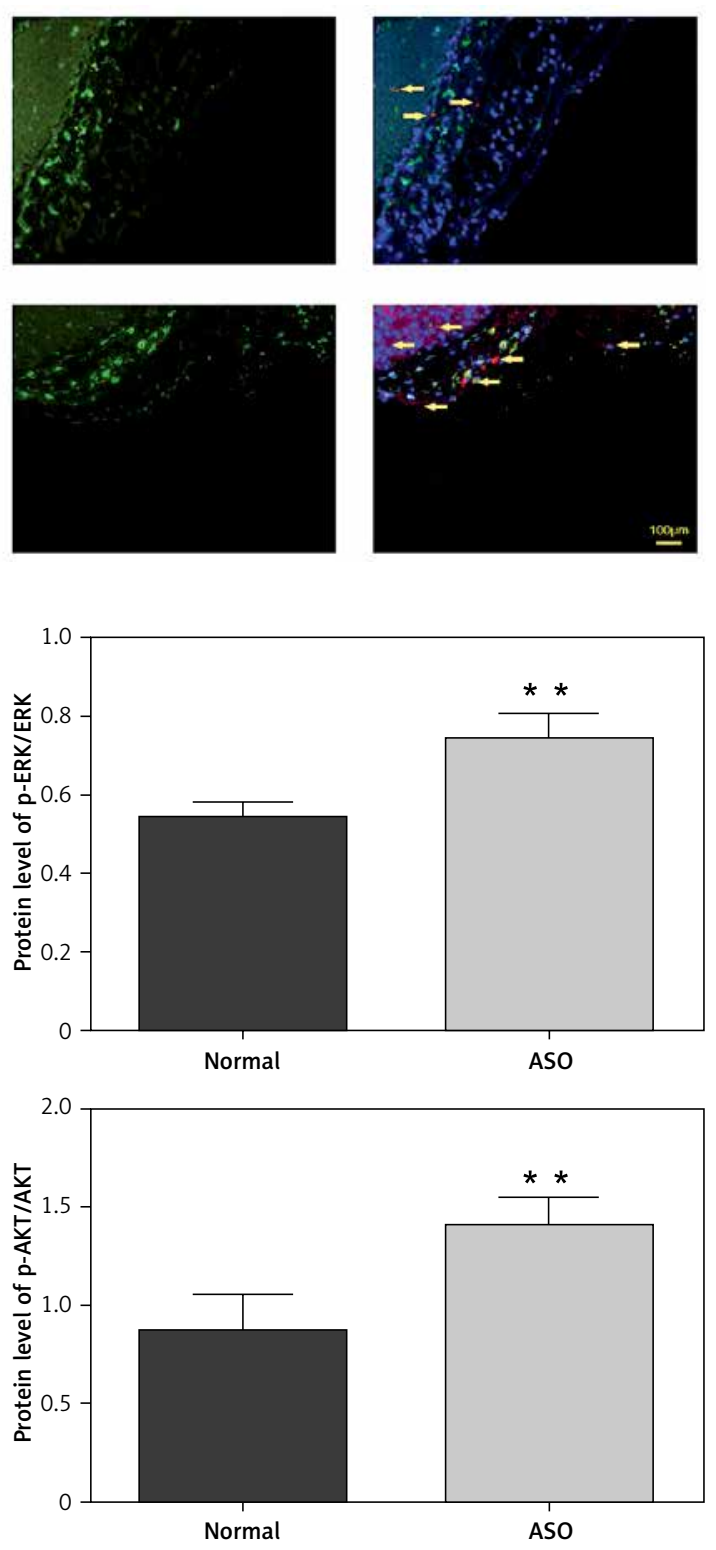

Figure 1. Characteristics of miR-21 expression and AKT and ERK $1 / 2$ activation in ASO artery. A - The miR-21 expression level in normal and ASO artery, determined by qRT-PCR. B - miR-21 expression in different layers of ASO arteries, determined by qRT-PCR. C - Immunohistochemistry of miR-21 expression level in ASO sections, miR-21 (red) and $\alpha$-actin (green). D - Tissues from ASO artery were resolved by SDS-PAGE and protein levels were detected using anti-AKT antibody (1 : 5000), anti-p-AKT antibody (1: 2000), anti-ERK antibody (1: 5000), anti-p-ERK antibody $(1: 2000)$ and anti- $\beta$-actin antibody $(1: 10000)$. Statistical analysis was performed according to Material and methods (data are reported as mean \pm SD, Student $t$-test, ${ }^{* *} p<0.01,{ }^{*} p<0.05,{ }^{\# \#} p<0.01$ ) 
sion level of miR-21 in miR-21 mimic and miR-21 mimic + AKT or ERK1/2 inhibitor groups. It was found that HASMC migrated through uncoated porous filters $(8.0 \mu \mathrm{m})$ in the Transwell migration assay. As shown in Figure $2 \mathrm{~B}$, transfection of miR21 mimics significantly accelerated the migration rate of HASMCs co-incubated with miR-21 mimics compared to that of the control group. To investigate whether miR-21 could promote cells growth, proliferation of cells in which miR-21 had been enhanced by the BrdU incorporation assay was examined. As expected, the cells transfected with miR-21 mimics showed a significantly increase in the number of BrdU-positive cells compared to the control group ( $p<0.05$; Figure $2 \mathrm{C}$ ). To explore the roles of the AKT and ERK $1 / 2$ signaling pathway in miR-21-induced proliferation and migration, HASMCs were transfected with miR-21 mimics and were then exposed to AKT or ERK inhibitors (Figure $2 \mathrm{~A}$ ). It was found that co-treatment with miR-21 mimics and AKT or ERK inhibitor could attenuate the miR-21-induced proliferation and migration of HASMCs ( $p<0.05$; Figure 2 B). Inhibition of the AKT or ERK activity might protect HASMC from miR-21-induced aberrant growth and motility. The results indicated that miR-21 overexpression promoted HASMC migration and proliferation. Furthermore, inhibition of AKT or ERK activity attenuated the aforementioned miR21-induced effects.

\section{Down-regulation of AKT and ERK1/2 expression altered miR-21 induced cell shape alteration in HASMCS}

To explore whether miR-21 promoted increasing expression of HASMCs $\alpha$-actin, miR-21 mimics were transfected into HASMCs to induce overexpression of miR-21. Cell shape alteration was determined with a confocal microscope and the cell elongation ratio was examined by measuring the longest cord and the longest axis perpendicular to it. Cell cytoskeleton was visualized by immunocytostaining of smooth muscle $\alpha$-actin (Figure $3 \mathrm{~B})$. Results from cell elongation assays showed that the cells co-treated with miR-21 mimics and AKT or ERK inhibitor could attenuate the miR-21induced high elongation ratio of $\operatorname{HASMCS}(p<$ 0.05 ; Figures $3 \mathrm{~A}$ ). The results indicated that miR21 not only played an important role in promoting HASMCs $\alpha$-actin, but also altered HASMCs' shape via AKT and ERK signaling pathways.

\section{Discussion}

Although miRNAs have been well established as key mediators in multiple physiological and pathological processes of cardiovascular disease, the mechanism remains unclear. Recent stud- ies concluded the pathology of ASO from clinical tests, but only ASO in relatively late stages could be detected based on the pathology known. As a result, finding a biomarker for detecting ASO in earlier stages is important. It is well accepted that miR-21 may play important roles in diverse vascular remodeling [24]. Wang et al. reported that the level of miR-21 significantly increased in ASO arteries [11]. However, signaling pathways involved in the regulation of proliferation and migration of VSMCs in ASO remain unclear. In this study, we firstly determined that the inhibition of AKT and ERK1/2 expression could attenuate miR-21-induced proliferation and migration of HASMCs. It was also found that the expression level of miR21 significantly increased in the ASO arteries compared to that in the normal arteries. This finding was supported by quantitative RT-PCR and immunofluorescence staining results (Figures $1 \mathrm{~A}, \mathrm{C}$ ). Expression of miR-21 was detected in different layers of the ASO artery, and it was found to be located in the media, combined with smooth muscle cells. The results indicated that aortic smooth muscle cells might partially contribute to the overexpression of miR-21 in the pathogenesis of ASO.

Activation of AKT and activation of ERK1/2 kinase are two important pathways regulating metabolism, proliferation and migration of VSMCS [25]. In the process of atherosclerosis, cell death and survival of VSMCs correlate with atherosclerotic plaque instability and stability, respectively [26]. Regulatory molecules including ERK1/2, AMP kinase, PI3K, Akt and mTOR can maintain cellular quality of lipoprotein metabolisms, such as of obesity and atherosclerosis [27]. In the present study, it was observed that activation of $\mathrm{P}-\mathrm{AKT}$ and p-ERK1/2 was significantly increased in the ASO artery, consistent with previous studies which found that angiogenesis was induced by miR-21 by the activation of AKT and ERK $1 / 2$ signaling pathways [28]. The present study added new proof to show that the expression of $p-A K T$ and $p$ ERK1/2 was involved in the pathogenesis of ASO.

To further study the role of miR-21 in HASMC motility, miR-21 mimics that can specifically stimulate the expression of miR-21 in HASMC were used and quantitative RT-PCR was used to detect the expression level of miR-21. It was found that overexpression of miR-21 significantly accelerated HASMC migration and proliferation rates, consistent with the previous study [13]. To explore the function of the AKT and ERK1/2 signaling pathways in miR-21-induced proliferation and migration, HASMCs were transfected with miR-21 mimics and were then exposed to AKT or ERK inhibitor. It was found that the inhibition of AKT or ERK $1 / 2$ could block both miR-21-induced migration and proliferation of HASMCS. The results confirmed 
miR-21 regulates vascular smooth muscle cell function in arteriosclerosis obliterans of lower extremities through AKT and ERK1/2 pathways

$\begin{array}{lllll}\text { A } \\ \text { miR-21 mimic } & - & + & + & + \\ \text { U0126 } & - & - & + & - \\ \text { AKT inhibitor } & - & - & - & +\end{array}$

p-ERK

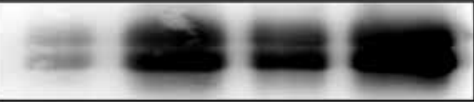

ERK

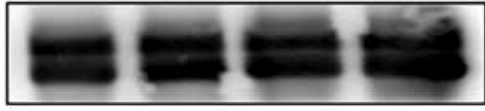

p-AKT

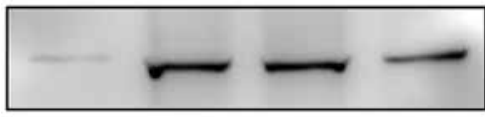

AKT
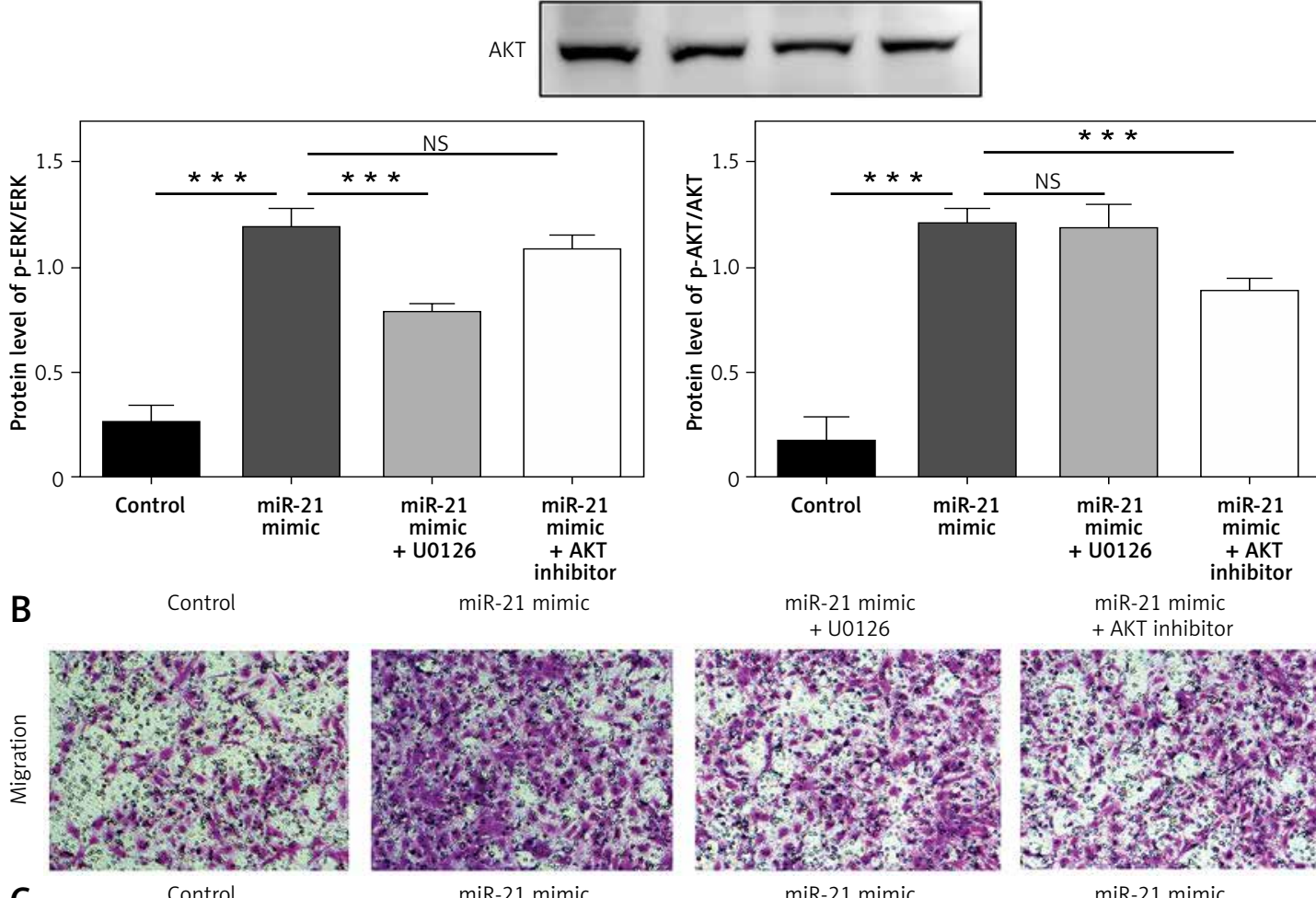

miR-21 mimic

miR-21 mimic

+ U0126

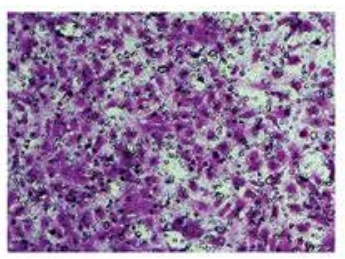

miR-21 mimic
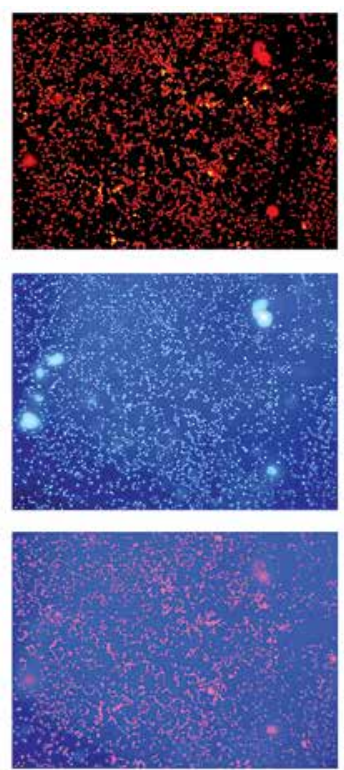

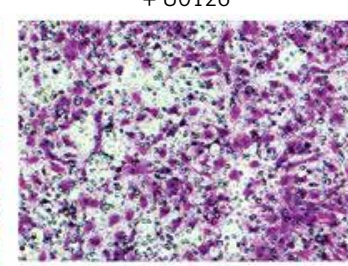

miR-21 mimic

+ U0126
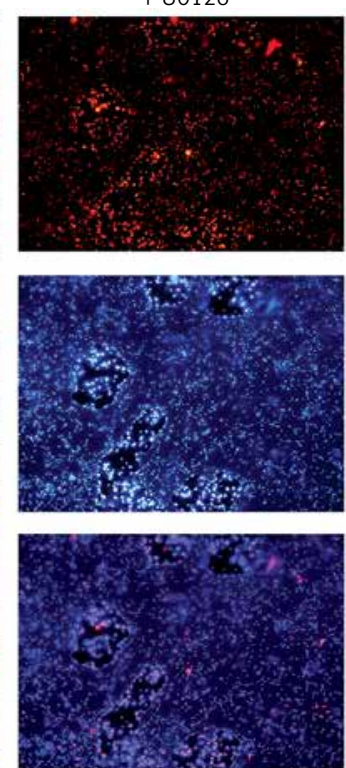

miR-21 mimic + AKT inhibitor

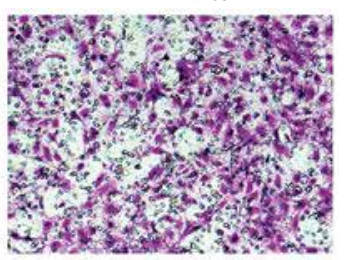

miR-21 mimic

+ AKT inhibitor
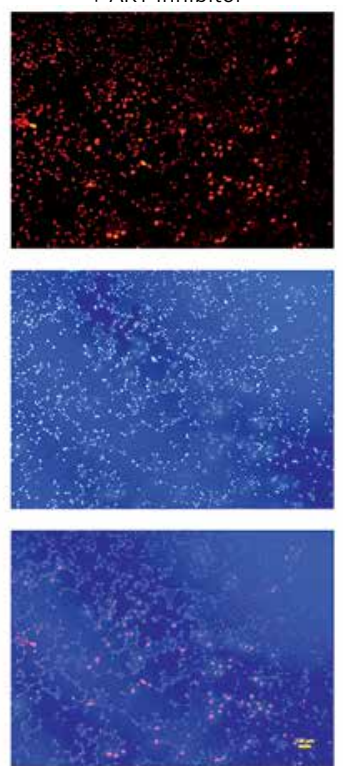

Figure 2. Effects of AKT and ERK1/2 expression inhibited miR-21-induced proliferation and migration of HASMC. A - Western blot showed suppression of AKT and ERK activation by using AKT inhibitor and ERK inhibitor U0126. B - HASMC migration rate was examined after transfection with miR-21 mimic and co-treatment with AKT or ERK1/2 inhibitor, determined by wound healing assay and Transwell assay. C - Representative images illustrate that BrdU expression was significantly decreased in miR-21 mimic + AKT or ERK1/2 inhibitor groups. Statistical analysis was performed according to Material and methods (data are reported as mean \pm SD, Student $t$-test, ${ }^{* *} p<0.01,{ }^{\#} p<0.05,{ }^{\# \#} p<0.01$ ) 
A

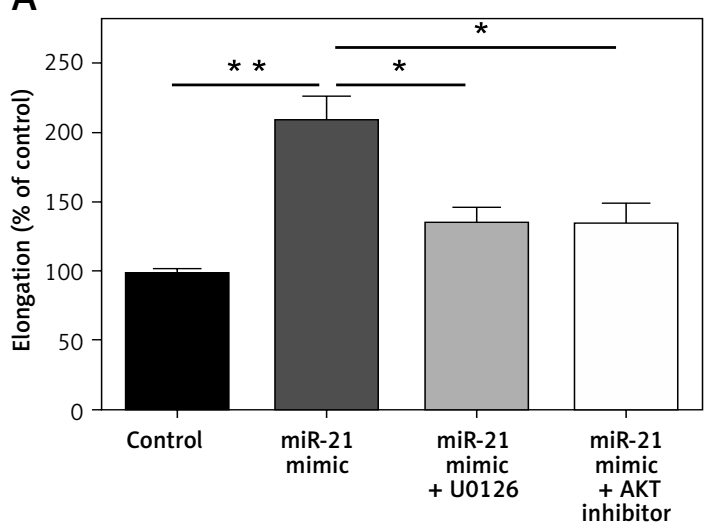

B

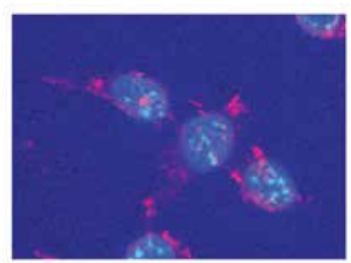

miR-21 mimic

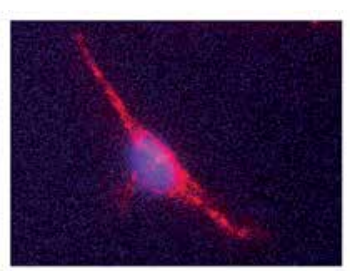

miR-21 mimic + U0126

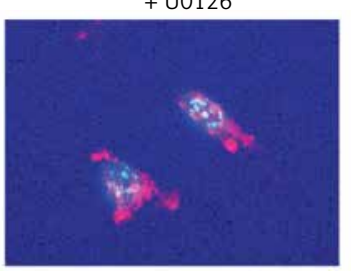

miR-21 mimic + AKT inhibitor

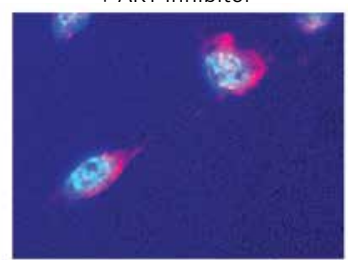

Figure 3. MiR-21 altered cell shape in HASMCs via AKT and ERK1/2 signaling pathway. A - Immunostaining was used to alter the cell shape followed by examination of cell elongation ratio. B - Results of smooth muscle $\alpha$-actin (red), and cell nuclear stain (blue) under confocal microscope demonstrated that overexpression of miR-21 altered cell shape and co-treatment with ERK or AKT inhibitor could be used to reverse the effect. Statistical analysis was performed according to Material and methods (data are reported as mean $\pm \mathrm{SD}$, Student $t$-test, ${ }^{* *} p<0.01$, $\left.{ }^{\#} p<0.05,{ }^{\# \#} p<0.01\right)$

that miR-21 functioned partially by activating AKT and ERK $1 / 2$ expression and restored the motility of HASMCs.

Recently studies demonstrated that miR-21 could regulate the smooth muscle $\alpha$-actin protein rate and altered cell shape change by directly targeting TPM1 in HASMCs [11]. It is still unclear, however, if cell shape changes in HASMCs correlate with AKT or ERK signaling pathways. In this study, it was proposed that miR-21 modulated the cytoskeleton by reducing the contraction of actin filaments via activating AKT and ERK $1 / 2$ expression. The cells co-treated with miR-21 mimics and AKT or ERK inhibitor could attenuate the miR-21-induced high elongation ratio of HASMCs. In summary, miR-21 not only played an important role in promoting HASMCs' $\alpha$-actin, but also altered HASMCs' cell shape via AKT and ERK signal pathways.

In conclusion, in the present study, we demonstrated that the expression of miR-21 in HASMCs is an aspect worth considering for the treatment of cardiac diseases. We found that miR-21 was significantly increased in the ASO process and promoted the motility of HASMCs. Irregular function of HASMCs serves a critical role in arteriosclerosis and ASO. The characteristics of miR-21 in the present study allow great progress in the understanding of the pathogenesis of ASO. The inhibition of AKT or ERK activity could attenuate the
miR-21-induced artery dysfunction effects as well as altering HASMCs' cell shape. The research provided a potential therapeutic approach and new targets for preventing and treating ASO in earlier stages.

\section{Acknowlegments}

Shuichuan Huang and Tuo Xu contributed equally.

This work was supported by Guangdong Science and Technology Plan Projects (2017A020215124), Guangdong Natural Science Foundation (2018A030313607) and President Foundation of Nanfang Hospital, Sothern Medical University (2016B023), Zhanjiang Science and Technology Plan Projects (2017B01120).

\section{Conflict of interest}

The authors declare no conflict of interest.

\section{References}

1. Vasto S, Candore G, Balistreri CR, et al. Inflammatory networks in ageing, age-related diseases and longevity. Mech Ageing Dev 2007; 128: 83-91.

2. Blankenberg S, Barbaux S, Tiret L. Adhesion molecules and atherosclerosis. Atherosclerosis 2003; 170: 191-203.

3. Steg PG, Bhatt DL, Wilson PW, et al. One-year cardiovascular event rates in outpatients with atherothrombosis. JAMA 2007; 297: 1197-206. 
4. Criqui MH, Vargas V, Denenberg JO, et al. Ethnicity and peripheral arterial disease: the San Diego Population Study. Circulation 2005; 112: 2703-7.

5. Diehm C, Allenberg JR, Pittrow D, et al. Mortality and vascular morbidity in older adults with asymptomatic versus symptomatic peripheral artery disease. Circulation 2009; 120: 2053-61.

6. Spronk S, Bosch JL, den Hoed PT, Veen HF, Pattynama PM, Hunink MM. Intermittent claudication: clinical effectiveness of endovascular revascularization versus supervised hospital-based exercise training-randomized controlled trial. Radiology 2009; 250: 586-95.

7. Reinecke $H$, Unrath $M$, Freisinger $E$, et al. Peripheral arterial disease and critical limb ischaemia: still poor outcomes and lack of guideline adherence. Eur Heart J 2015; 36: 932-8.

8. Kim J, Zhang L, Peppel K, et al. Beta-arrestins regulate atherosclerosis and neointimal hyperplasia by controlling smooth muscle cell proliferation and migration. Circ Res 2008; 103: 70-9.

9. Hao H, Gabbiani G, Bochaton-Piallat ML. Arterial smooth muscle cell heterogeneity: implications for atherosclerosis and restenosis development. Arterioscler Thromb Vasc Biol 2003; 23: 1510-20.

10. Schachter M. Vascular smooth muscle cell migration, atherosclerosis, and calcium channel blockers. Int J Cardiol 1997; 62: S85-90.

11. Wang M, Li W, Chang GQ, et al. MicroRNA-21 regulates vascular smooth muscle cell function via targeting tropomyosin 1 in arteriosclerosis obliterans of lower extremities. Arterioscler Thromb Vasc Biol 2011; 31: 2044-53.

12. Zhang C. MicroRNomics: a newly emerging approach for disease biology. Physiol Genom 2008; 33: 139-47.

13. Ji R, Cheng Y, Yue J, et al. MicroRNA expression signature and antisense-mediated depletion reveal an essential role of MicroRNA in vascular neointimal lesion formation. Circ Res 2007; 100: 1579-88.

14. Matsumoto T, Hwang PM. Resizing the genomic regulation of restenosis. In.: Am Heart Assoc 2007.

15. Jagavelu K, Tietge UJ, Gaestel M, Drexler H, Schieffer B, Bavendiek U. Systemic deficiency of the map kinase-activated protein kinase 2 reduces atherosclerosis in hypercholesterolemic mice. Circ Res 2007; 101: 1104-12.

16. Matsuzawa A, Ichijo H. Molecular mechanisms of the decision between life and death: regulation of apoptosis by apoptosis signal-regulating kinase 1 . J Biochem 2001; 130: 1-8.

17. Matsuzawa A, Ichijo H. Redox control of cell fate by MAP kinase: physiological roles of ASK1-MAP kinase pathway in stress signaling. Biochim Biophys Acta 2008; 1780: 1325-36.

18. Li JY, Liu CP, Shiao WC, et al. Inhibitory effect of PDGF$\mathrm{BB}$ and serum-stimulated responses in vascular smooth muscle cell proliferation by hinokitiol via up-regulation of p21 and p53. Arch Med Sci 2018; 14: 579.

19. Zhuang J, Peng W, Li H, et al. Inhibitory effects of vinpocetine on the progression of atherosclerosis are mediated by Akt/NF-kappaB dependent mechanisms in apoE/-mice. PLoS One 2013; 8: e82509.

20. Motoshima H, Goldstein BJ, Igata M, Araki E. AMPK and cell proliferation-AMPK as a therapeutic target for atherosclerosis and cancer. J Physiol 2006; 574: 63-71.

21. Zhai C, Cheng J, Mujahid H, et al. Selective inhibition of $\mathrm{PI} 3 \mathrm{~K} / \mathrm{Akt} / \mathrm{mTOR}$ signaling pathway regulates autophagy of macrophage and vulnerability of atherosclerotic plaque. PloS One 2014; 9: e90563.
22. Zhan Y, Kim S, Izumi Y, et al. Role of JNK, p38, and ERK in platelet-derived growth factor-induced vascular proliferation, migration, and gene expression. Arterioscler Thromb Vasc Biol 2003; 23: 795-801.

23. $\mathrm{Xu}$ Q. Biomechanical-stress-induced signaling and gene expression in the development of arteriosclerosis. Trends Cardiovasc Med 2000; 10: 35-41.

24. Thum T, Catalucci D, Bauersachs J. MicroRNAs: novel regulators in cardiac development and disease. Cardiovasc Res 2008; 79: 562-70.

25. Jiang BH, Jiang G, Zheng JZ, Lu Z, Hunter T, Vogt PK. Phosphatidylinositol 3-kinase signaling controls levels of hypoxia-inducible factor 1. Cell Growth Differ 2001; 12: 363-70.

26. Ambrose JA, D’Agate DJ. Classification of systemic therapies for potential stabilization of the vulnerable plaque to prevent acute myocardial infarction. Am J Cardiol 2005; 95: 379-82.

27. Meijer AJ, Codogno P. Signalling and autophagy regulation in health, aging and disease. Mol Aspects Med 2006; 27: 411-25.

28. Liu LZ, Li C, Chen Q, et al. MiR-21 induced angiogenesis through AKT and ERK activation and HIF-1alpha expression. PloS One 2011; 6: e19139. 